EPIDEMIC MEASLES IN CHICAGO IN 1983: SUSTAINED TRANS-

+ 510 mission IN THE PRESCHOOL POPULATION. Michael L. Bennish, Paul M. Arnow, Sandra A. Doveik1s, and Marc . University of Chicago, Wyler Children's

\section{Ber The}

(ER) the first week of 1983 prompted active ER-based survelllance for other cases. Between $1 / 4 / 83$ and $8 / 12 / 83,107$ suspect cases were identifled, and 85 had diagnostic tests for measles. Measles was proven in 54 cases by $>4$-fold rise in CF or HI titers in paired serum specimens or by virus isolation from nasopharyngeal secretions. Fever, rash, and cough, coryza, or conjunctivitis secretions. Fever, rash, and cough, cor of atients with negative were present in $84 \%$ of cases and $57 \%$ of patients with negative measles diagnostic tests. All 54 measles pattents were native born, and 52 were black. $23(43 \%)$ were $<16$ months old, and 41 $38 \%(8 / 21)$ for children 55 years old and $3 \%(4 / 116)$ for persons 5 to 25 years old $(p<.001)$. Only 7 patients were in a day care or sch. The oxclusion of children school. The lacking proof of measles lmmuntate significantly from that following school exclusion (1.4/wk) or end of the school year (2.0/wk). Measles antibodies were meas
by ELISA in serum specimens collected in the ER about 1 year by ELISA in serum specimens collected in the ER about 1 year were seronegative. With current immunization policies and practices a pool of susceptibles can develop in pre-school children of the inner city that is capable of supporting a sustained improved strategies for immunization of this group.
A TOTAL POPULATION STUDY OF OUT-OF-HOSPITAL CARDIO-

513 RESPIRATORY ARREST IN CHILDREN. P.C. Couture, P.H. Children's Hospital, Dept. of Pediatrics, Boston.

While the epidemiology of cardiorespiratory arrest in adults has been extensively studied, this issue in children has remained relatively unexplored. We reviewed all cases of out-of-hospital cardiorespiratory arrest in resident Boston children to identify their incidence, causes, demography, and contribution to total childhood mortality. Case-specific data were collected from emergency room (ER) logs and medical records of Boston hospitals. Death certificate analysis provided total mortality data.

Between 1978 and 1982, 270 out-of-hospital arrests occurred in resident children ages 1 month to 20 years. $155(57 \%)$ were not transported to a hospital. $115(43 \%)$ underwent resuscitation in hospital ER's. $19(17 \%)$ of these were resuscitated and survived hospitalization. During this time period a total of 480 deaths occurred in this same population of children. Therefore, 251 (52\%) of these deaths occurred out-of-hospital. Of the arrests treated in $\mathrm{ER}^{\prime}$ 's, $46 \%$ were in infants, 35\% between 1 and 15 years, and $19 \%$ between 16 and 20 years of age. $30 \%$ were due to trauma, $28 \%$ to SIDS; $62 \%$ were sudden with no predisposing condition $20 \%$ had an identifiable cause ongoing for 12 hours or more. This study suggests than an emphasis on hospital based therapies will not influence a large portion of childhood deaths.
Efforts to reduce mortality in children must recognize the major contribution made by out-of-hospital events. The nature of this mortality suggests that preventive and pre-hospital interventions will be of primary importance.

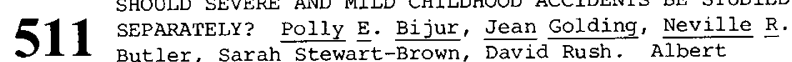

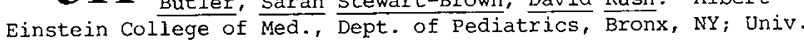
of Bristol, Dept. of Child Health, Bristol, UK.

The distinction between accidents of varying severity is rareThe distinction between accidents of varying severity is rarely made in studies of the accident process. If different social and behavioral processes lead to severe and mild accidents, or
if there is more error in the report of mild accidents, inference will be biased. Social and behavioral characteristics of 11,966 British children from birth to five and mother's reports of accidents requiring medical attention were analyzed. Severe accidents were those resulting in hospitalization; mild accidents were those treated on an out-patient basis. The report of severe were those treated on an oute than mild accidents $56 \%$ of the seaccidents was more reliable than mild accidents: in the medical vecord, versus $14 \%$ of the mild accidents. Simultaneous multiple record, versus $14 \%$ of the mild accidents. Simultaneous multiple regressions revealed diffexent patterns of association: mild accidents were most significantly associated with male sex, followed by mother's depression, young age, poor housing, liberal attitudes, non-neurotic and antisocial behavior. Severe accidents were most strongly associated with older siblings, mother's youth, child's antisocial behavior, mother's depression, breath holding, hearing problems, male sex and number of moves. The patterns of associations suggest that there is both differential reporting of accidents of varying severity and different processes involved. we conclude that inference about accident processes will be more valid if severe and mild accidents are analyzed separately.

THE EPIDEMIOLOGY OF CHILDHOOD APPENDICITIS: A CASE-

512 CONTROL STUDY, Jean D. Brender, Noel S. Weiss, Tom Bergman). Children's Orthopedic Hospital and Medical Center and University of Washington Department of Epidemiology, Seattle.

To better understand the etiology of childhood appendicitis, case-control study of this disease was conducted among 135 children who underwent an appendectomy between November 1980 and December 1982. Two hundred twelve comparison children, matched December 1982. Two hundred twelve comparison children, matched to these cases by age, sex, and admission date, were selected from the orthopedic and short stay services of the same hospital. Children having one or more first degree relatives with a posit-
ive history of appendectomy for appendicitis were 1.2 times more ive history of appendectomy for appendicitis were 1.2 times mor
likely to develop appendicitis $(p>0.10)$ than children without this history. No significant increase in risk for appendicitis was noted in children having parents with a positive history of appendectomy for appendicitis, but children with one or more siblings affected by this disease were 9.5 time more likely to siblings affected by this disease were 9.5 time more likely to have appendicitis $(p<0.05)$. As the proportion of family members with a positive history of appendectomy increased, the risk of childhood appendicitis increased $(p=0.002)$. A significant protective effect was demonstrated between intake of cereal fiative risk: $0.53, p<0.05$ ). A significant "dose-response" effect was also noted between increasing levels of whole-grain cereal intake and decreasing risk of appendicitis $(p=0.024)$. The roles of birthweight, growth indices, allergies, laxatives, and constipation were also investigated, and none of these and constipation were also investigated, and none
RISK FACTORS ASSOCIATED WITH HOSPITAL ACQUISITION

514 (HA) OF ROTAVIRUS INFECTION (RVI) ON AN INFANT WARD. Penelope H. Dennehy and Georges Peter. Brown University and Rhode Island Hospital, Department of Pediatrics, Providence, RI.

To assess the incidence and risk factors of HA of RVI on an infant ward, as defined by the presence of RV antigen in stool examined by ELISA (Abbott), children admitted with community-acquired gastroenteritis (CA GI), those with diarrhea developing quired gastroenterision tients and selected uninfected controls (C) were enrolled during tients and selected uninfected controls (C) were enrolled during
a 6 mos. period. Of 583 prospectively followed admissions, 23 of $60 \mathrm{CA}$ GI were due to RV. In spite of enteric isolation of GI cases, 36 additional infants developed HA RVI; the attack rate of HA RVI totalled 68 , ranging monthly from 1.0 to $14 \%$. Of HA cases, 26 of $36(728)$ cases had no room contact with an RVI case, in comparison to only 7 of $31 \mathrm{C}(22 \%) \quad(p<.0002)$, and 9 of 36 (258) HA RVI were in isolation rooms for unrelated reasons, indicating that isolation did not prevent $\mathrm{HA}$ of RVI. The major dicating that isolation did not prevent $\mathrm{HA}$ of RVI. The major
risk factor was season; $698(16 / 23)$ of $\mathrm{CA}$ and $75 \%(27 / 36)$ NA cases occurred during the late winter and early spring. 15 of $36 \mathrm{HA}(428)$ and 9 of $31 \mathrm{C}(298)$ shared the same houseofficer; similarly, 28 of $36(78 \%)$ and 25 of 31 (818), respectively, shared a nurse. Mean duration of hospitalization, 25.9 d in HA was significantly longer than that of $21.8 \mathrm{~d}$ in $\mathrm{c}(\mathrm{p}=0.04)$. These findings indicate that HA of rotavirus is common in spite of enteric precautions; results in prolonged hospitalization and, thus, potentially preventable costs; and indicate the need for further studies to elucidate modes of transmission.

BATERIOLOGIC EXAMINATION OF GASTRIC ASPIRATE (GA)

515 AS A SCREEN FOR NEONATAL SEPSIS. Jane M. Dempsey and William J. Cashore, Brown

Hospital, Dept. Of Ped., Providence, R. I.

213 of 508 consecutive neonatal ICU admissions (Jan-June,
983 ) had gastric aspirate cultures for prolonged rupture of membranes ( $>18 \mathrm{hrs}$ ), maternal fever, or respiratory distress at birth. To evaluate the use of GA cultures as a screen for neonatal sepsis or pneumonia, we compared GA culture results with clinical outcome, autopsy findings, radiologic diagnosis of pneumonia, and results of cultures from other sites. 18 of the 213 infants had confirmed sepsis or pneumonia, with positive and negative GA cultures distributed as shown in the table:

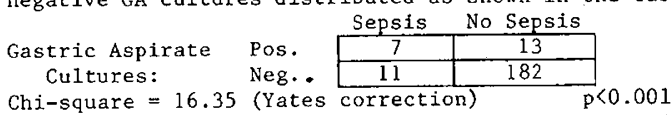

7 of $18 \mathrm{GA}$ cultures $(39 \%)$ in newborns with serious infections, including 6 of $8(75 \%)$ in newborns with bacteremia, were positive for the specific pathogen found at other sites. 13 of 195 GA cultures (7\%) gave "false positive" results in newborns $195 \mathrm{GA}$ cultures (7\%) gave fof 182 of 195 with no subsequent evidence of neonatal infection. infants ( $93 \%$ ) evaluated for infection because of perinat factors, but with no subsequent supporting evidence for
perinatal infection, had negative GA cultures. GA is easily obtained for culture and microscopic examination at birth. Bacteriologic examination of GA has a low rate of faise positive results and may permit early recognition of bacterial pathogens in some newborns with serious perinatal infections. 